\title{
On-line multidimensional scaling programs with interactive graphical display
}

\author{
J. J. CHANG* \\ Bell Laboratories \\ Murray Hill, New Jersey 07974
}

The beginnings of a system of interactive multidimensional scaling programs with real-time display of the graphical output have been established on the Honeywell DDP-224 computer. Two programs have been completed: (1) MDPREF-a computer program for multidimensional analysis of preference data-has been converted from the GE-635 to run interactively on the DDP.224 computer. Its solution is printed from a typewriter, and the configuration of stimuli are displayed on a scope in two-dimensional view. (2) ROTATE-an on-line rotation program-enables the user to rotate the configuration in three dimensions within a higher dimensional space.

As of today, more than a score of multidimensional scaling (MDS) programs are stored in cabinets, on tapes, or on permanent disks. The idea of establishing a systematic MDS program library has been around for a long time, nevertheless the objectives were never immediate. We always look far ahead to the day when, as formulated by Roger N. Shepard: "A coherent system of computer programs and associated graphical output will be implemented where the researcher could sit at a console directing the flow of aralyses via an on-line interactive process."

As a first step towards this goal, we have launched our initial test on a Honeywell DDP-224 computer with a MDS program named MDPREF, which stands for multidimensional analysis of preference data.

Multidimensional scaling generally refers to a set of methods for deriving spatial representation from psychological data. Most of the data are measures of similarity or dissimilarity of pairs of stimuli or measures of preference or other dominance relations. Since the solutions of most MDS programs (except INDSCAL) are invariant under orthogonal rotations, there of ten exists a problem, i.e., the problem of how to rotate the solution to a set of psychologically interpretable dimensions. At times. orthogonal rotations can be easily performed by hand; however, it becomes virtually impossible when more than three dimensions are involved. To solve the rotation problem, an on-line rotation program has been devised which is capable of carrying out the rotations in higher dimensions.

At present, two programs have been implemented on the DDP-224: (1) MDPREF, which performs the MDS analysis of preference data. has its solution printed from a typewriter and the configuration displayed on a scope

*I am indebted to J. D. Carroll, M. V. Mathews, and P. D. Bricker for their helpful and valuable suggestions. in a two-dimensional view. (2) ROTATE, an on-line rotation program, which enables the user to rotate a configuration in three dimensions within a higher dimensional space.

\section{The DDP Computer}

The Honeywell DDP-224 digital computer is a 24-bit machine with 32,000 words of memory and a cycle time of 1.9 microsec. Its peripheral storage devices include one Control Data magnetic tape transport, two Control Data disk drives capable of storing 57 million bits of data on each disk pack, a card reader, and numerous display devices connected to the computer, including a large cathode ray tube (CRT).

Programs written in Fortran or DAP can be punched on cards or stored on tape. Either device can serve as input to the DDP Fortran compiler or DAP assembler. Object programs are stored on disk and may be transferred to tapes, so the object program can be loaded from either the disk or the tape. The input data can be read in from the card reader, the tape, or the typewriter. For on-line display, a black box with four knobs, four toggle switches, and two pushbuttons serve as input devices. A picture of the control panel, the typewriter, and the scope is shown in Fig. 1.

\section{MDPREF -MULTIDIMENSIONAL ANALYSIS OF PREFERENCE DATA}

MDPREF is a computer program written in Fortran IV. It was designed to carry out the multidimensional analysis of paired-comparison or other preference data described by Carroll \& Chang (1964) and also Carroll (1972). MDPREF takes as input several paired-comparison data matrices, one for each $S$, from which a rectangular $S$ by Stimuli matrix is derived. The Eckart-Young procedure of factorization is then applied to this matrix to yield the best configuration of stimulus points and $S$ vectors (in some specified number of dimensions), such that the projections of stimuli on each S's vector are as similar as possible to the order of preference judgment expressed by the S. (For a full description of the program, see Chang \& Carroll, 1968.) The solution is displayed on the scope. showing the locations of stimulus points and the Ss' vectors on the plane of a pair of dimensions at a time. At present. the program can compute solutions in up to five dimensions.

\section{ROTATION}

The objectives and usefulness of the rotation program are manifold. Mainly it is designed to solve the problem of finding the correct orientation for some MDS solutions. There are many statistical methods for 

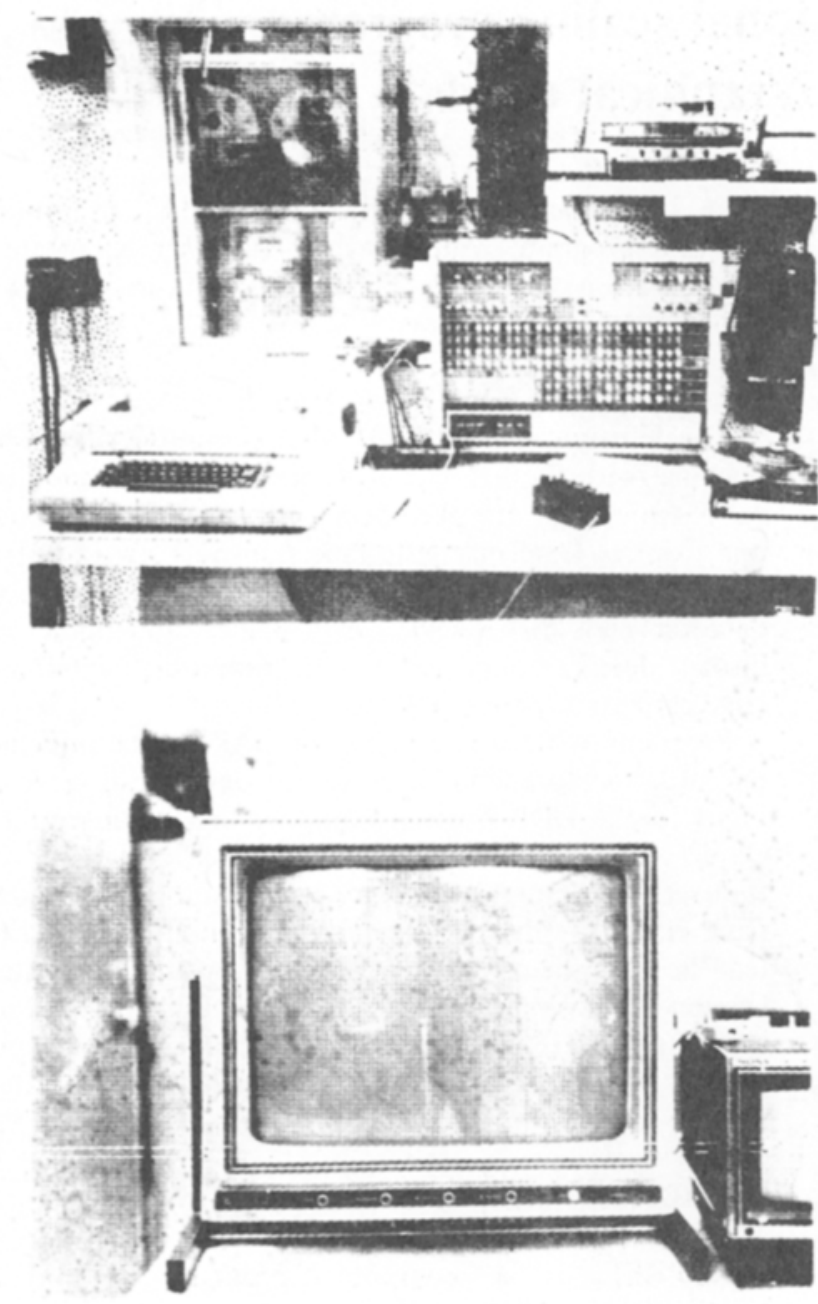

Fig. 1. Control panel with typewriter and cathode ray tube display.

orienting dimensions (such as the principal axis method of extracting dimensions which account for the successively greater amount of variance in data, the Varimax, or other analytic rotation procedures and various configuration matching procedures). Nevertheless, in many cases, especially in pattern search, the meaningful relationship among points can only be perceived and interpreted through intuition, common sense, and possibly by some cognitive processes. Such subjective methods may at times prove to be superior to the objective statistical methods when the problem is to rotate the solution to psychologically interpretable axes. A search of this type, however, is virtually impossible without a rapid interactive facility for rotation and display. The rotation program brings searching for structure in more than three dimensions into the realm of practicality.

At present, the rotation program is capable of only orthogonal rotations, and it can be used to accomplish the following objectives: (A) To rotate a configuration of points to find interpretable dimensions. (B) To rotate the configuration to match some other solution. (C) To rotate the points around an arbitrary vector which can be a vector representing a known physical property obtained from PROFIT, which is a computer program for fitting properties by a regression procedure. The rotations can also be done in the plane which is normal to the arbitrary vector. (D) To search for hidden geometric patterns or patterns of clustering. An experiment was carried out to test if it was possible to find a two-dimensional pattern embedded in a five-dimensional space. The procedure and result of the experiment will be described later. (E) It has been found that the rotation program is very helpful in crystallography, where some known crystal structures are embedded in a higher dimensional space. By calling various crystallographic directions normal to the plane of projection, one is able to rotate the structure until the desired pattern could be found.

\section{"ROTATE"-A COMPUTER PROGRAM WHICH PERFORMS ON-LINE ROTATION OF POINTS}

ROTATE utilizes the real-time interactive computer graphics routines written by Michael Noll (1968) to draw lines, characters, and dots on the scope.

\section{Input Features}

Given the coordinates of $\mathrm{N}$ points in $\mathrm{K}$ dimensions ohtaincd from some MDS solution, ROTATE takes a set of any three dimensions out of the total set and displays the points on the scope with horizontal and vertical axes. The three numbers corresponding to the three dimensions are input from the typewriter. The first number represents the first dimension of the given set and corresponds to the horizontal axis, and the second number is shown as the vertical axis. The third axis is invisible but pointing in the direction of the viewer. The input devices are mounted on a black box, as shown in

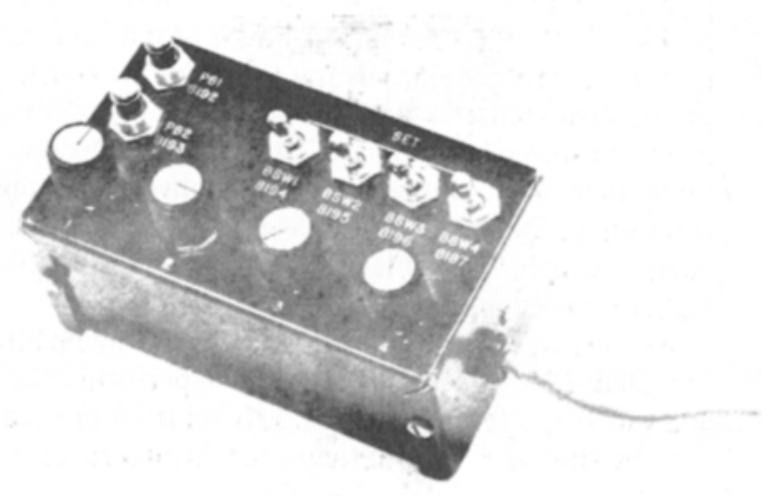

Fig. 2. The black box. 
Fis. 2. Knobs 1.2. and 3 command the rotation of the poumts around the lirst. sciond. or the third axis, respectively. Knob 4 rotates the points in the plane normal to an arbitrary vector (will be described later). There are two pushbuttons. one of which will return the configuration to the beginning of the current set of three dimensions: hence. all the rotations done from that time are wiped out. The other pushbutton will record the configuration at the time the button is pressed and type out a message to request a new set of dimensions to be typed in. The recording is accomplished by writing on tupe the transformation matrix and the transformed coordinates of points. The four switches provide the option of: (A) reading in an arbitrary vector (in three dimensions only): (B) displaying the plane normal to the arhitrary vector; (C) printing either characters or dots at the lucation of the points: (D) returning the configuration to the very beginning. i.e., to the first set of three dimensions.

\section{Description of Rotation}

To rotate a point $P=(x, y)$ about the origins in a plane through Angle $\theta$ to its new position. $\mathrm{p}^{*}=\left(\mathrm{x}^{*}, \mathrm{y}^{*}\right)$, the equations describing the rotation are

$$
\begin{aligned}
& x^{*}=x \cos \theta+y \sin \theta \\
& y^{*}=-x \sin \theta+y \cos \theta
\end{aligned}
$$

In matrix form, they can be written as $\mathrm{P}^{*}=\mathrm{TP}$, where $\mathrm{T}$ is a transformation matrix.

For the purpose of rotating the points about each axis dinstinctly and to take into account the characteristics of the computer design, the program computes the transformed coordinates as follows:

(A) The program reads the three knobs simultaneously. The reading on the knob is from 0 to 4095. The angle change in a plane is computed by the following equation:

$$
\Delta \theta=[\mathrm{KNOB}(\text { previous })-\mathrm{KNOB}(\text { present })] / 8 \text {. }
$$

(B) The delta transformation matrix is computed by

$$
\Delta T=\prod_{i=1}^{3} T_{i}
$$

where $T_{i}$ is the transformation matrix for Plane $j k$ where $\mathrm{i} \neq \mathrm{j} \neq \mathrm{k}$ (e.g., $\mathrm{T}_{1}$ is the transformation matrix for Plane 23). The reason for deriving $\Delta T$ this way is that when only one knob is turned. the desired rotation should be restricted to around that particular axis corresponding to the knob. However. the interference of an unpredictable amount of noise in knobs made it impossible to do so. Consequently, it has been found that by multiplying the $\left(T_{1}\right) s$ in this manner. the rotations are perceived to be distinctly about the chosen axis.
The $\left(T_{i}\right)$ s are defined as follows:

$$
\begin{aligned}
& T_{1}=\left|\begin{array}{ccc}
1 & 0 & 0 \\
0 & \cos \theta_{1} & \sin \theta_{1} \\
0 & -\sin \theta_{1} & \cos \theta_{1}
\end{array}\right| \\
& T_{2}=\left|\begin{array}{ccc}
\cos \theta_{2} & 0 & \sin \theta_{2} \\
0 & 1 & 0 \\
-\sin \theta_{2} & 0 & \cos \theta_{2}
\end{array}\right| \\
& T_{3}=\left|\begin{array}{ccc}
\cos \theta_{3} & \sin \theta_{3} & 0 \\
-\sin \theta_{3} & \cos \theta_{3} & 0 \\
0 & 0 & 1
\end{array}\right|
\end{aligned}
$$

$\theta_{\mathrm{i}}, \mathrm{i}=1,3$. is the angle change when rotating about the ith axis. by:

(C) The overall transformation matrix $\mathrm{T}$ is updated

$$
T(\text { new })=(\Delta T) T(\text { old })
$$

(D) $\mathrm{T}$ is orthogonalized by the Gram-Schmidt procedure. This step is introduced to keep $T$ from gradually becoming nonorthogonal by accumulation of rounding errors.

(E) New coordinates are computed by

$$
P^{*}=T(\text { new }) P
$$

This two-step process, in which $\mathrm{P}^{*}$ is always calculated as an orthogonal transformation of the original $\mathrm{P}$, is also designed to prevent accumulation of rounding errors.

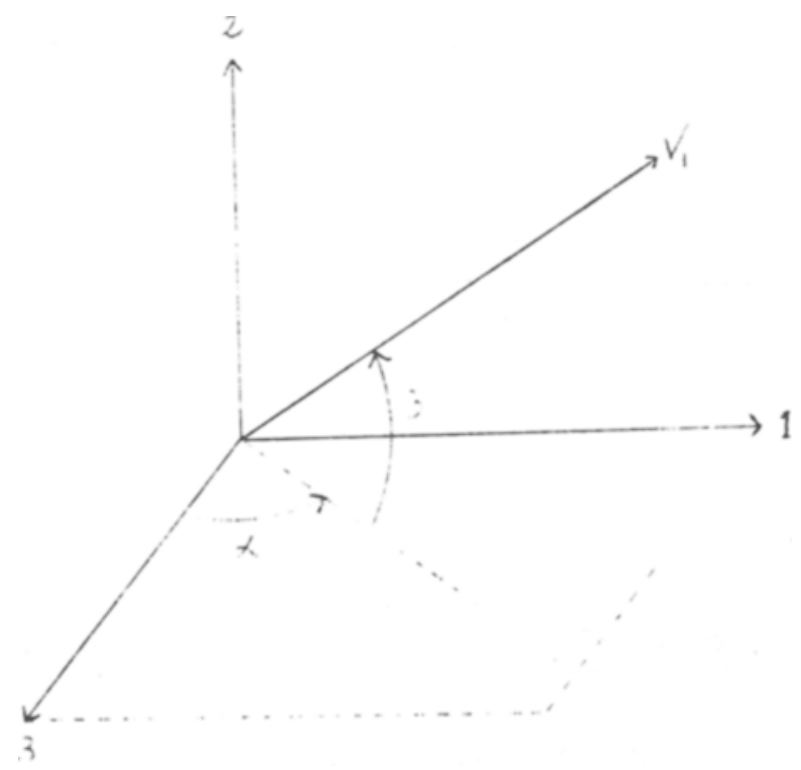

Fig. 3. Using Angles a and, 3 to define the position of Vector $V$, with respect to Axes 1,2 , and 3 . 

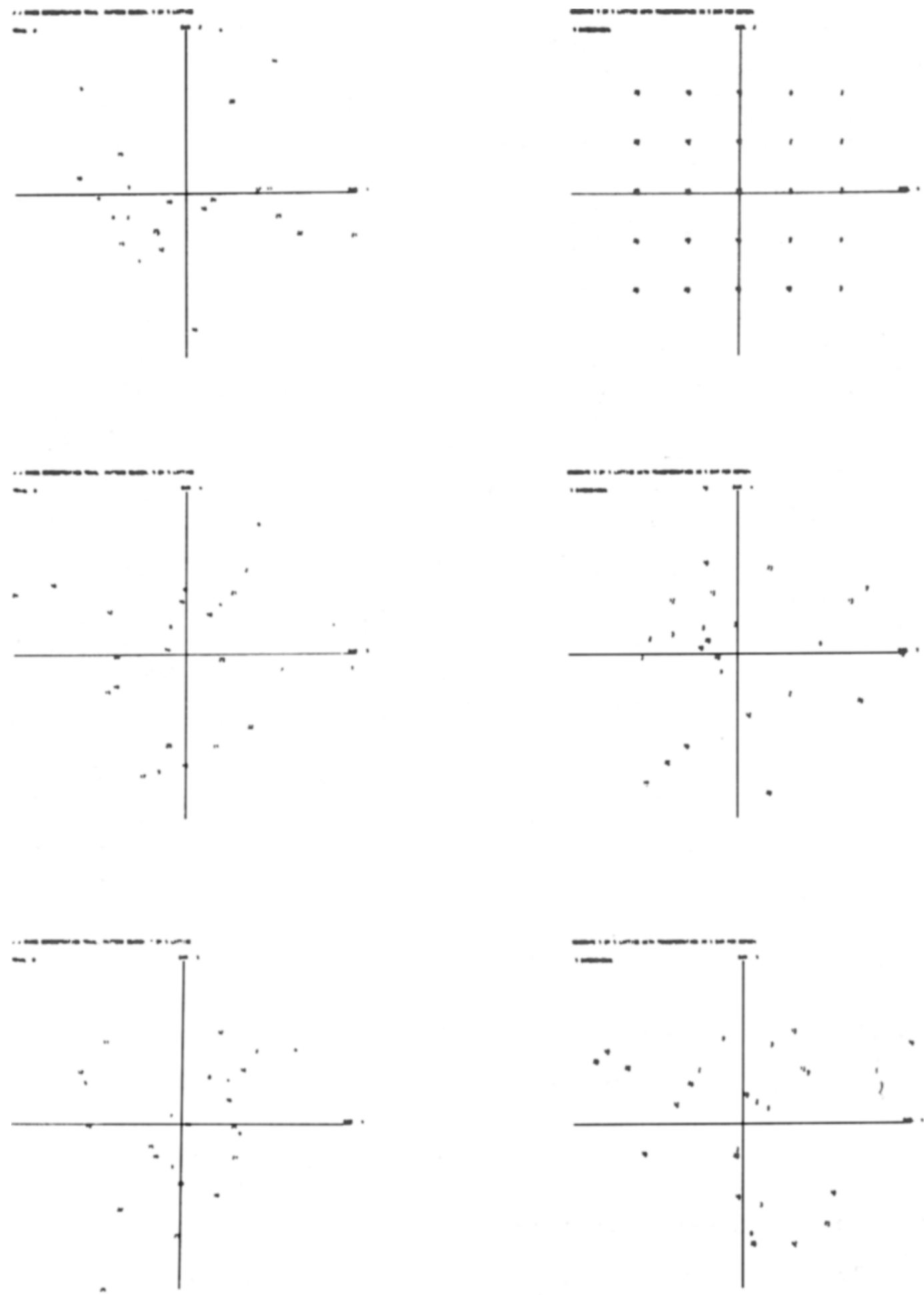

Fig. 4. Configuration of 25 points used in pattern search experiment. Right: The original configuration. Left: Transformed configuration (after a random orthogonal transformation).

Rotation About an Arbitrary Vector

An arbitrary vector may be read in from the typewriter. The input can either be three direction numbers (coordinates) or two angles. $\alpha$ and $\beta$. where $\alpha$ is the angle away from the third axis in the 1,3 plane and $\beta$ is the angle away from and perpendicular to the 1,3 plane (se. Fig. 3).

To rotate the points about an arbitrary vector. first let 
Vhe the new orthogunal basis

$$
v=\left[\begin{array}{l}
v_{1} \\
v_{2} \\
v_{3}
\end{array}\right]
$$

where $V_{1}=\left[n_{1}, n_{2}, n_{3}\right], n_{1}, n_{2}$, and $n_{3}$ are the direction numbers typed in by the user. If Angles $\alpha$ and $\beta$ are used: $n_{1}=\cos (\beta) \sin (\alpha): n_{2}=\sin (\beta): n_{3}=\cos (\beta) \cos (\alpha)$. To find Vectors $V_{2}$ and $V_{3}$, let $V_{K}=(1.0 .0)$

$$
\text { Compute } \begin{aligned}
\mathrm{V}_{2} & =\operatorname{CROSS}\left(\mathrm{V}_{\mathrm{K}} \cdot \mathrm{V}_{1}\right) \\
\mathrm{V}_{3} & =\operatorname{CROSS}\left(\mathrm{V}_{1}, \mathrm{~V}_{2}\right)
\end{aligned}
$$

where CROSS $(A . B)=\left(a_{2} b_{3}-a_{3} b_{2}\right)_{i}+\left(a_{3} b_{1}-a_{1} b_{3}\right)_{j}$ $+\left(a_{1} b_{2}-a_{2} b_{1}\right)_{k}$.

While the points are rotated around the arbitrary vector. they can either be viewed in the original set of coordinates or be projected onto the normal plane. The changes in angles of rotation are read from KNOB 4. Let $\mathrm{U}$ be the transformation matrix on rotation. $\mathrm{U}$ is computed in the same way as $T$ in Eqs. 1 to 5. To project the points in the original basis, let $Z=V^{\prime} U V$ and $X^{*}=Z T X$, where $X^{*}$ are the rotated coordinates and $T$ is the old transformation matrix. To change the projection to the new basis, let $Z^{*}=V Z, X^{*}=\left(Z^{*} T\right) X$. To return again to the original basis, let $Z^{* *}=V^{\prime} Z^{*} . X^{*}$ $=(\mathrm{Z} * \mathrm{~T}) \mathrm{X}$.

\section{EXPERIMENT ON PATTERN SEARCH}

An informal experiment was designed to investigate how difficult it would be for people to find a two-dimensional configuration embedded in a five-dimensional space by the rotation process described above. The five-dimensional configuration was generated as follows: a set of 25 points in a 5 by 5 lattice pattern was constructed in the 1,2 plane, and for the remaining three dimensions. random numbers were used as coordinates. A random orthogonal transformation was applied to this 5 by 25 matrix. The resulting configuration is shown on the left in Fig. 4, along with the original configuration
The $S$ was first shown a plot of the 5 by 5 lattice design and wat then presented with the random configuration on the scope. The S was instructed that the objective of the "game" was to make the lattice appear on the scope. The technique and mechanism of rotation were explained. The typewriter and the black box were at the S's command. Eighteen Ss participated in the experiment, and the time limit was $60 \mathrm{~min}$. Nine $S \mathrm{~s}$ failed to find the pattem; nire Ss, five of whom had had experience with IIDS involving rotation of points, found the lattice in times ranging from 15 to $60 \mathrm{~min}$.

Most of the Ss who succeeded used similar strategies, i.e., they always had the same plane displayed while varying the third dimension. One interesting point is that some Ss reported that they were trying to figure out some mathematical mapping strategy and that it was virtually impossible to follow the points in the five dimensions. Four other people who succeeded said that they concentrated on the feedback of the perceived movements of the points in the displaved plane.

\section{CONCLUSION}

Further work is being planned to explore ways of extracting more information in detecting clusters and outlyers. Work will continue on the conversion of other MDS programs and the sequencing of programs, so that the solution of one can serve as input to another. Eventually, all programs and the on-line solutions will be stored on permanent disks or tape, and the user sitting at the console following a few instructions will be able to perform several different MDS analyses on the original or the derived (solution or rotated solution from a previous run) set of data.

\section{REFERENCES}

Carroll, J. D., \& Chang, J. J. Non-parametric multidimensional analysis of paired comparison data. Paper presented at the joint meeting of the Psychometric Society and the Psychonomic Society, Niagara Falls, New York, October 1964.

Carroll. J. D. Individual differences and multidimensional scaling. In $R$. N. Shepard. A. K. Romney, and S. Nerlove (Eds.), Multidimensional scaling: Theory and applications in the behavioral sciences. New York: Seminar Press, 1972.

Chang, J. J., \& Carroll, J. D. How to use MDPREF, a computer program for multidimensional analysis of preference data. Unpublished paper, Bell Laboratories, 1968.

Noll, $M$. Software package for real-time interactive computer graphics on the Honeywell DDP-224 computer. Unpublished paper, Bell Laboratories, 1968 . 